\title{
Steady-state density functional theory for thermoelectric effects
}

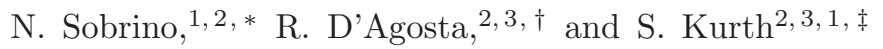 \\ ${ }^{1}$ Donostia International Physics Center, Paseo Manuel de Lardizabal 4, E-20018 San Sebastián, Spain \\ ${ }^{2}$ Nano-Bio Spectroscopy Group and European Theoretical Spectroscopy Facility (ETSF), \\ Departamento de Física de Materiales, Universidad del País Vasco UPV/EHU, \\ Avenida de Tolosa 72, E-20018 San Sebastián, Spain \\ ${ }^{3}$ IKERBASQUE, Basque Foundation for Science, Maria Diaz de Haro 3, E-48013 Bilbao, Spain
}

(Dated: September 25, 2019)

\begin{abstract}
The recently proposed density functional theory for steady-state transport (i-DFT) is extended to include temperature gradients between the leads. Within this framework, a general and exact expression is derived for the linear Seebeck coefficient which can be written as the sum of the KohnSham coefficient and an exchange-correlation contribution. The formalism is applied to the singleimpurity Anderson model for which approximate exchange-correlation functionals are suggested for temperatures both above and below the Kondo temperature. A certain structural property of the exchange-correlation potentials in the Coulomb blockade regime allows to recover an earlier result expressing the Seebeck coefficient in terms of quantities of equilibrium density functional theory. The numerical i-DFT results are compared to calculations with the numerical renormalization group over a wide range of temperatures finding a reasonable agreement while i-DFT comes at a much lower computational cost.
\end{abstract}

\section{INTRODUCTION}

During the last years, thermoelectric materials have attracted the attention of both experimental and theoretical research interest due to their wide variety of possible technological applications. $\frac{1-3}{-3}$ These applications aim mainly at the conversion of waste heat into an electrical current through the Seebeck effect or at cooling through the Peltier effect $\underline{\underline{4}}$ An efficient thermocouple for heat-toelectricity conversion exhibits a low thermal conductance as well as a large electrical conductance and Seebeck coefficient $\stackrel{4}{=}$ In standard bulk materials there are intrinsic difficulties in disentangling the phonon and electron contributions to the transport coefficients: improving the electrical conductance usually increases the thermal conductance and reduces the Seebeck coefficient. Systems of reduced dimensionality are a promising alternative since a more accurate and independent control of the diverse factors dominating the thermoelectric efficiency appears possible.$^{\frac{5}{2}}$ The path towards molecular electronics and the discovery of two dimensional materials further expand the scope of potential materials for thermoelectric applications $\underline{\underline{6}-\underline{8}}$ However, it still remains a difficult problem to predict which new materials (bulk, layered or heterostructure) are most apt as thermoelectric devices.

The theory of thermoelectric energy conversion draws from many sources,$\underline{-14}$ but recently, Density Functional Theory (DFT) has acquired a prominent role in almost any material modeling $: \frac{75,16}{1}$ This popularity is mainly due to two factors: (i) relatively simple approximations to the so-called exchange-correlation (xc) functional, the central quantity of DFT, lead to reasonably accurate results for many material properties and (ii) DFT is numerically very efficient. For the description of (electronic or thermal) transport, DFT is typically combined with the Landauer-Büttiker (LB-DFT) formalism, $\underline{6}-8.17$ However, formally this approach is incomplete since it treats electrical transport as a scattering problem of non-interacting electrons and uses equilibrium DFT for an inherently out-of-equilibrium system. A proper DFT framework to describe electronic transport is timedependent DFT (TDDFT) $\stackrel{18}{=}$ In TDDFT, one may view steady-state transport as the long-time limit of a system initially in equilibrium and driven out of equilibrium by application of an external bias. In the steady state, this approach will then lead to equations structurally identical to the LB-DFT equations but with the external bias augmented by a dynamical xc correction $\underline{\underline{19}} \underline{\underline{24}}$ As a consequence, the connection between quantities of the real system and those calculated within the LB-DFT framework is typically nontrivial and strong corrections may appear $\stackrel{25,26}{\longleftarrow}$

More recently, an alternative DFT framework to describe steady-state electronic transport, dubbed i-DFT, has been proposed $\frac{25}{2}$ i-DFT directly focusses on the steady state and is designed to give both the density and the current of a (DC) biased molecular junction 25 However, it does not make any statements on the time evolution towards such a steady state. Nevertheless, just as TDDFT, it also leads to xc corrections to the bias. So far, functionals for these xc bias corrections have been constructed for model systems $\stackrel{25,27,28}{=}$ In particular, i-DFT allows to describe the single impurity Anderson model (SIAM) both in the Coulomb Blockade (CB) as well as in the Kondo regime 27

The standard form of i-DFT assumes that the device and the leads are thermally equilibrated. In this work we generalize the formalism to finite temperature gradients between the leads which allows one to use i-DFT as a highly efficient method to calculate the Seebeck coefficient. Paying special attention to the temperature dependence of the i-DFT equations we derive: a) an exact expression for the linear Seebeck coefficient for any system and any regime in terms of pure i-DFT quantities 
and b) a pair of xc functionals for the SIAM with explicit dependence on the thermal gradient $\Delta T$ between the leads which are exact for $T \gg \gamma$, i.e., for weakly coupled leads. We also model the effect of the broadening due to the couplings in the functionals and we equip our functionals to describe the linear Seebeck coefficient in the Kondo regime in an accurate way.

\section{I-DFT FOR THERMOELECTRIC EFFECTS}

We consider the typical transport setup where a central region, e.g., a single molecule or a quantum dot, is coupled to a left (L) and a right $(\mathrm{R})$ electrode. The system is driven out of equilibrium by applying a DC bias $V$ across the junction and we are interested in the resulting steady-state current $I$. The recently suggested i-DFT framework for steady-state transport ${ }^{25}$ is based on a one-to-one mapping between, on the one hand, the density $n(\mathbf{r})$ in the central region and the steady current $I$ through it and, on the other hand, the external potential $v(\mathbf{r})$ in the same region and the bias $V$ across it. In the original formulation, both left and right leads are kept at the same temperature $T$ which enters the formalism as an external parameter only. Here we propose an extension of i-DFT to include a temperature difference between the leads. This thermal gradient creates an electronic current which can be compensated by a bias in an open circuit setup and thus allows to study the Seebeck effect. For simplicity, we symmetrically apply both a bias $V$ as well as a temperature difference $\Delta T$ between the two leads, i.e., we have $V_{\alpha}= \pm V / 2$ and $T_{\alpha}=T \pm \Delta T / 2$ where $\alpha=L, R \stackrel{29}{2}$ Of course, now both $T_{L}$ and $T_{R}$ (or, equivalently, $T$ and $\Delta T$ ) enter as parameters into the formalism. If we make the (physically reasonable) assumption that the density in the central region and the current are continuously differentiable at $\Delta T=0$, the original i-DFT $\operatorname{proof}^{25}$ of the one-to-one correspondence between "densities" and "potentials" can directly be applied to our situation and we can formulate the i-DFT theorem for leads at different temperatures (see also Ref. 30 ).

Theorem: For any pair of finite temperatures $T_{\alpha}$ in the leads, there exists a one-to-one correspondence between the pair of "densities" $(n, I)$ and the pair of "potentials" $(v, V)$ in a finite (and gate dependent) region around zero voltage $V$ and zero thermal gradient $\Delta T$.

It is important to note that the proof of the theorem goes through for any form of the interaction, particularly also for the non-interacting case. As usual, in order to establish a Kohn-Sham (KS) scheme, we have to assume non-interacting representability, i.e., that the same densities $(n, I)$ of an interacting system (with potentials $(v, V))$ can also be obtained as densities of a noninteracting system with potentials $\left(v_{s}, V_{s}\right)$. Following the standard KS procedure, we define the Hartree-exchangecorrelation (Hxc) gate potential as $v_{\mathrm{Hxc}}[n, I]=v_{s}[n, I]-$ $v[n, I]$ and the xc bias as $V_{\mathrm{xc}}[n, I]=V_{s}[n, I]-V[n, I]$. The self-consistent coupled KS equations for the density and the current then are

$$
\begin{gathered}
n(\mathbf{r})=2 \sum_{\alpha=L, R} \int \frac{\mathrm{d} \omega}{2 \pi} f_{\alpha}\left(\omega-V_{\alpha, s}\right) A_{\alpha, s}(\mathbf{r}, \omega) \\
I=2 \sum_{\alpha=L, R} \int \frac{\mathrm{d} \omega}{2 \pi} f_{\alpha}\left(\omega-V_{\alpha, s}\right) s_{\alpha} \mathcal{T}(\omega)
\end{gathered}
$$

where $f_{\alpha}(x)=1 /\left(e^{x / T_{\alpha}}+1\right)$ is the Fermi function (for lead $\alpha$ ), $s_{L / R}= \pm$, and $V_{\alpha, s}=s_{\alpha}\left(V+V_{\mathrm{xc}}\right) / 2$. $A_{\alpha, s}(\mathbf{r}, \omega)=\left\langle\mathbf{r}\left|\mathcal{G}(\omega) \Gamma_{\alpha}(\omega) \mathcal{G}^{\dagger}(\omega)\right| \mathbf{r}\right\rangle$ is the partial $\mathrm{KS}$ spectral function with the KS Green's function $\mathcal{G}$ and the broadening matrix $\Gamma_{\alpha}$ of lead $\alpha$. Finally, the transmission function is given by $\mathcal{T}(\omega)=\operatorname{Tr}\left[\mathcal{G}(\omega) \Gamma_{L}(\omega) \mathcal{G}^{\dagger}(\omega) \Gamma_{R}(\omega)\right]$.

Eqs. (1) and (2) have the same structure as the original i-DFT equations ${ }^{25}$ with the exception that the temperature difference $\Delta T$ between the two leads enters explicitly both in the Fermi functions $f_{\alpha}$ and in the functionals for $v_{\mathrm{Hxc}}$ and $V_{\mathrm{xc}}$. In the following we use exactly this property to derive an exact expression for the Seebeck coefficient in terms of i-DFT quantities.

The Seebeck coefficient is defined as that bias which has to be applied to compensate a small temperature difference between the leads such that no current flows. Formally it can be written as

$$
S=\left.\frac{d V}{d \Delta T}\right|_{\substack{V=0 \\ \Delta T=0}}=\left.\frac{d I / d \Delta T}{d I / d V}\right|_{\substack{V=0 \\ \Delta T=0}} .
$$

Both the numerator and the denominator of Eq. (3) can be calculated directly from Eq. (2). The denominator is nothing but the zero-bias conductance $G$ which can be expressed as 25

$$
G=\left.\frac{d I}{d V}\right|_{\substack{V=0 \\ \Delta T=0}}=\frac{G_{s}}{1-\left.G_{s} \frac{\partial V_{x c}}{\partial I}\right|_{\substack{V=0 \\ \Delta T=0}}}
$$

where we have defined the $\mathrm{KS}$ zero bias conductance

$$
G_{s}=-\int \frac{\mathrm{d} \omega}{2 \pi} f^{\prime}(\omega) \mathcal{T}(\omega) .
$$

where $f^{\prime}(x)=\mathrm{d} f / \mathrm{d} x$ with the Fermi function taken at the temperature $T=T_{L}=T_{R}$.

Similarly, the numerator of Eq. (3) can be calculated as

$$
\begin{aligned}
\left.\frac{d I}{d \Delta T}\right|_{\substack{V=0 \\
\Delta T=0}} & =\int \frac{\mathrm{d} \omega}{2 \pi}\left[f^{\prime}(\omega)\left(\frac{\omega}{T}+\frac{d V_{x c}}{d \Delta T}\right)\right] \mathcal{T}(\omega) \\
& =\frac{L_{s}-\left.G_{s} \frac{\partial V_{x c}}{\partial \Delta T}\right|_{\substack{V T=0 \\
\Delta T=0}}}{1-\left.G_{s} \frac{\partial V_{x c}}{\partial I}\right|_{\substack{V=0 \\
\Delta T=0}}}
\end{aligned}
$$

where we have defined

$$
L_{s}=\frac{1}{T} \int \frac{\mathrm{d} \omega}{2 \pi} f^{\prime}(\omega) \omega \mathcal{T}(\omega) .
$$

In deriving Eq. (6) we have expanded out the total derivative,

$$
\frac{\mathrm{d} V_{x c}}{d \Delta T}=\frac{\partial V_{x c}}{\partial \Delta T}+\frac{\partial V_{x c}}{\partial I} \frac{d I}{d \Delta T}+\int \mathrm{d}^{3} r \frac{\delta V_{x c}}{\delta n(\mathbf{r})} \frac{d n(\mathbf{r})}{d \Delta T},
$$


and used the fact that for $I=0$ (i.e., $V=0$ and $\Delta T=0$ ) the last term vanishes because $V_{\mathrm{xc}}[n, I=0]=0$.

Combining Eqs. (4) and (6), we then arrive at the following simple expression for the linear Seebeck coefficient

$$
S=S_{s}-\left.\frac{\partial V_{x c}}{\partial \Delta T}\right|_{\substack{\Delta V=0 \\ \Delta T=0}}
$$

where $S_{s}=\frac{L_{s}}{G_{s}}$ is KS Seebeck coefficient and the second term of Eq. (9) is the xc contribution. Eq. (9) is one of the central results of the present work. It is formally exact and expresses the Seebeck coefficient of a general interacting system solely in terms of i-DFT quantities. In practice, of course, one has to use approximations for the Hxc gate and the $\mathrm{xc}$ bias functionals. We will address the construction of such approximations for a model system in the next Section. We also point out that an expression similar to Eq. (9) was recently obtained in a standard DFT framework ${ }^{26}$ in the Coulomb Blockade (CB) regime. In the following Section, we will discuss the connection between the two approaches.

\section{SINGLE IMPURITY ANDERSON MODEL}

In this Section we consider the single-impurity Anderson model (SIAM) which also in previous works ${ }^{25-27}$ has been used as a first model for the development of approximate i-DFT functionals. The SIAM describes a single interacting impurity level (quantum dot) coupled to a left $(\mathrm{L})$ and right $(\mathrm{R})$ lead. The dot is described by the Hamiltonian

$$
\hat{H}^{\mathrm{dot}}=\sum_{\sigma} v \hat{n}_{\sigma}+U \hat{n}_{\uparrow} \hat{n}_{\downarrow}
$$

where $v$ is the on-site energy of the dot and $U$ is the interaction and $\hat{n}_{\sigma}$ is the operator for the density of electrons with spin $\sigma$ on the dot. The system is coupled to left and right featureless electronic leads described by frequencyindependent couplings $\Gamma_{\alpha}(\omega)=\gamma_{\alpha}($ with $\alpha=L, R)$, i.e., we work in the wide band limit (WBL). The leads are characterized by temperature $T_{\alpha}$ and may be subject to a DC bias $V_{\alpha}$ which we take to be symmetric, i.e., $V_{L}=-V_{R}=V / 2$.

If we want to study the system in an i-DFT framework, we need approximations for the $(\mathrm{H}) \mathrm{xc}$ functionals. The approximate functionals designed in previous work ${ }^{25.27}$ were restricted to the case of equal lead temperatures, $T_{L}=T_{R}$, and therefore we need to extend the construction to the more general case $T_{L} \neq T_{R}$.

Coulomb blockade regime - Following ideas used in earlier work ${ }^{25}$, we first aim to construct approximations for the xc functionals in the Coulomb blockade regime. We start by expressing both the density on and the current through the dot in terms of the many-body spectral func- tion $A(\omega)$ :

$$
\begin{aligned}
n= & \int \frac{\mathrm{d} \omega}{2 \pi}\left[\frac{2 \gamma_{L}}{\gamma} f_{L}\left(\omega-\mu-V_{L}\right)\right. \\
& \left.+\frac{2 \gamma_{R}}{\gamma} f_{R}\left(\omega-\mu-V_{R}\right)\right] A(\omega) \\
I= & \frac{2 \gamma_{L} \gamma_{R}}{\gamma} \int \frac{\mathrm{d} \omega}{2 \pi}\left[f_{L}\left(\omega-\mu-V_{L}\right)\right. \\
& \left.-f_{R}\left(\omega-\mu-V_{R}\right)\right] A(\omega)
\end{aligned}
$$

where $\gamma=\gamma_{L}+\gamma_{R}$ is the total broadening.

We want to use Eqs. (11) to reverse engineer the $(\mathrm{H}) \mathrm{xc}$ potentials of i-DFT, therefore we need a model for the many-body spectral function $A(\omega)$. As a starting point we use the exact spectral function of the isolated dot which is given by

$$
A_{0}^{\bmod }(\omega)=\left(1-\frac{n}{2}\right) \delta(\omega-v)+\frac{n}{2} \delta(\omega-v-U) .
$$

Using $A_{0}^{\bmod }(\omega)$ as model spectral function for the contacted dot and inserting it into Eqs. (11) leads to exactly the same expressions for density and current as one would obtain by working out the rate equations which are valid in the Coulomb blockade regime $\stackrel{9}{\underline{9}}$ Inserting Eq. (12) into Eqs. (11), the reverse-engineering for the Hxc gate and xc bias potentials can be done analytically. This follows by forming from Eqs. (11) the linear combinations $n+I / \gamma_{L}$ and $n-I / \gamma_{R}$ and realizing that the inversion of the resulting equations for the potentials $v \pm V / 2$ can be done exactly as in Refs. 31 and 32 . The resulting Hxc gate and $\mathrm{xc}$ bias potentials are

$$
\begin{gathered}
\tilde{v}_{\mathrm{Hxc}}=\frac{1}{2}\left(g\left(n,-I / \gamma_{R}, T_{R}\right)+g\left(n, I / \gamma_{L}, T_{L}\right)\right), \\
\tilde{V}_{\mathrm{xc}}=g\left(n,-I / \gamma_{R}, T_{R}\right)-g\left(n, I / \gamma_{L}, T_{L}\right),
\end{gathered}
$$

where we have defined

$$
g(n, x, T)=U+T \log \left(\frac{p+\sqrt{p^{2}-z y e^{-U / T}}}{y}\right)
$$

with $z=y-2, y=4 x+n$ and $p=n-1+2 x\left(1+e^{-U / T}\right)$. As mentioned above, Eqs. (13) are equivalent to reverseengineering the rate equations and therefore should be valid at high temperatures $T \gg \gamma$, i.e. in the parameter regime where the effect of temperature is much more important than the coupling to the leads.

For the construction of xc potentials which give reasonable approximations also in the regime of $T \sim \gamma$ we start by using a model spectral function 25 of the form (12) but with the delta functions replaced by Lorentzians $l_{\gamma}(\omega)=\frac{\gamma}{\omega^{2}+\gamma^{2} / 4}$ of width $\gamma=\gamma_{L}+\gamma_{R}$, i.e.

$$
A_{\gamma}^{\bmod }(\omega)=\left(1-\frac{n}{2}\right) l_{\gamma}(\omega-v)+\frac{n}{2} l_{\gamma}(\omega-v-U) .
$$




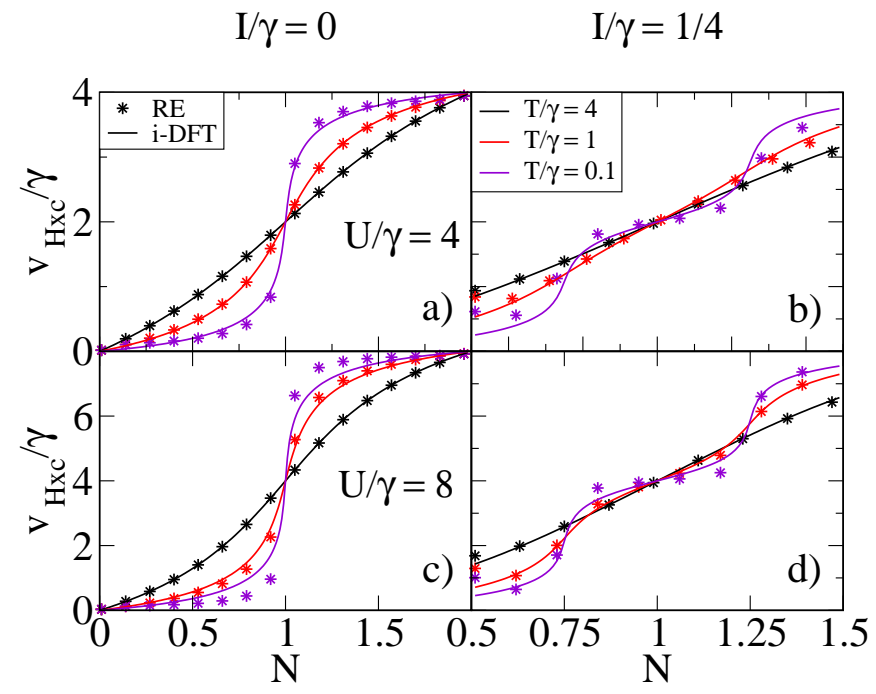

FIG. 1. Hxc potentials $\tilde{v}_{\mathrm{Hxc}}$ of the symmetrically coupled SIAM $\left(\gamma_{L}=\gamma_{R}=\gamma / 2\right)$ in the Coulomb blockade regime for different temperatures calculated by reverse-engineering (RE) compared to the parametrization of Eq. (16) Panels a) and b) are for $U / \gamma=4$, panels c) and d) for $U / \gamma=8$, while panels a) and c) are for zero current $I=0$ and panels b) and d) for $I / \gamma=1 / 4$.

For this model, the reverse-engineering cannot be carried out analytically (not even at $T=0$ ) but still can easily be done numerically. However, it is then desirable to have simple parametrizations of the resulting xc potentials. In Ref. 25 a simple parametrization for $T=0$ has been suggested. Since here we are interested in finite temperatures, a generalization is required. To construct such a parametrization we use the observation ${ }^{33}$ that both temperature $T$ and spectral broadening $\gamma$ lead to similar smearing out of step features which are present in the low-temperature and/or strongly correlated limit. Therefore we suggest a parametrization using the same analytic form as in Eqs. (13) but replacing the left and right temperatures $T_{L / R}$ by effective temperatures $T_{L / R}^{*}$, e.g.,

$$
\begin{gathered}
\tilde{v}_{\mathrm{Hxc}}=\frac{1}{2}\left(g\left(n,-I / \gamma_{R}, T_{R}^{*}\right)+g\left(n, I / \gamma_{L}, T_{L}^{*}\right)\right), \\
\tilde{V}_{\mathrm{xc}}=g\left(n,-I / \gamma_{R}, T_{R}^{*}\right)-g\left(n, I / \gamma_{L}, T_{L}^{*}\right) .
\end{gathered}
$$

The effective temperatures $T_{\alpha}^{*}$ we parametrize as

$$
T_{\alpha}^{*}\left(T_{\alpha}, \gamma\right)=\frac{T_{\alpha}^{2}+(\eta \gamma)^{2}+\eta \gamma T_{\alpha}}{T_{\alpha}+\eta \gamma}
$$

This parametrization is chosen in such a way that $T_{\alpha}^{*}\left(T_{\alpha}, \gamma \rightarrow 0\right)=T_{\alpha}$ and $\eta$ is a fit parameter for which we take the value $\eta=0.45$ in the Coulomb blockade regime. From now on, we always choose symmetric coupling of the leads, i.e. $\gamma_{L}=\gamma_{R}=\gamma / 2$. The quality of our parametrization can be appreciated in Fig. 1 where we

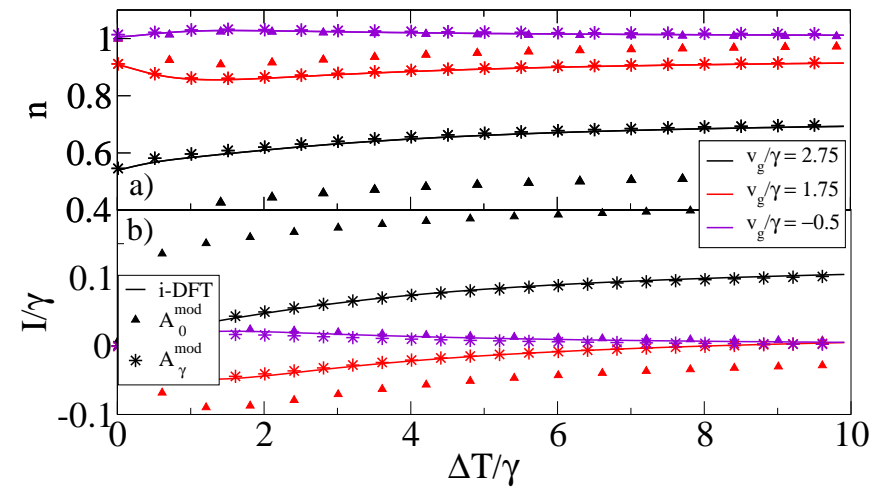

FIG. 2. Densities (panel a)) and currents (panel b)) as function of the temperature difference $\Delta T=T_{L}-T_{R}$ for $U / \gamma=5$, $T_{R}=0.1$ and $V=0$ for different gate voltages $v_{g}=v+\frac{U}{2}$. The i-DFT results using the xc potentials of Eq. (16) are compared with those obtained directly from Eq. (11) when using either the model spectral function $A_{0}^{\text {mod }}$ of Eq. (12) or $A_{\gamma}^{\text {mod }}$ of Eq. (15).

compare the model $\tilde{v}_{\mathrm{Hxc}}$ with the corresponding results of the reverse engineering (RE) from Eq. (15) for $U / \gamma=4$ (panels a) and b)) and $U / \gamma=8$ (panels c) and d)). We see that, at equilibrium $(I=0$, panels a) and $\mathrm{c})$ ), our parametrization reproduces the reverse-engineered Hxc potential very accurately for all the considered temperatures. Also at finite current $(I / \gamma=1 / 4$, panels b) and d)), our approach gives a reasonable parametrization of the reverse-engineered Hxc potential, although there are some differences at the borders of the domain for the lowest temperature.

As another check on the quality of our parametrization, in Fig. 2 we show the density and the electronic current induced by a temperature difference $\Delta T$ between the leads (at zero bias) as function of $\Delta T$ for different gate voltages $v_{g}=v+U / 2$. The i-DFT results are obtained using the xc potentials in Eqs. (16) and are compared to those obtained by the direct evaluation of Eqs. (11) with the model spectral functions of Eqs. (12) and (15), respectively. The agreement, especially for the latter case, is excellent.

An interesting structural property of the xc potentials of Eqs. (13) is that they are given as the sum of two pieces, each one depending only on the parameters (temperature and coupling) of one of the leads. For the couplings this has already been noted in Ref. 34. For the case of different temperatures in the leads, $T_{\alpha}=T \pm \Delta T / 2$, it can be easily shown that this structure leads to the xc contribution to the many-body Seebeck coefficient (Eq. (9)) of the form

$$
S_{x c}^{C B}=\left.\frac{\partial V_{x c}}{\partial \Delta T}\right|_{\substack{V=0 \\ \Delta T=0}}=\left.\frac{\partial v_{H x c}}{\partial T}\right|_{\substack{V=0 \\ \Delta T=0}} .
$$

While this result holds for any approximation with the structural property mentioned above, for the special case of the functionals of Eq. (13) it reduces exactly to the 


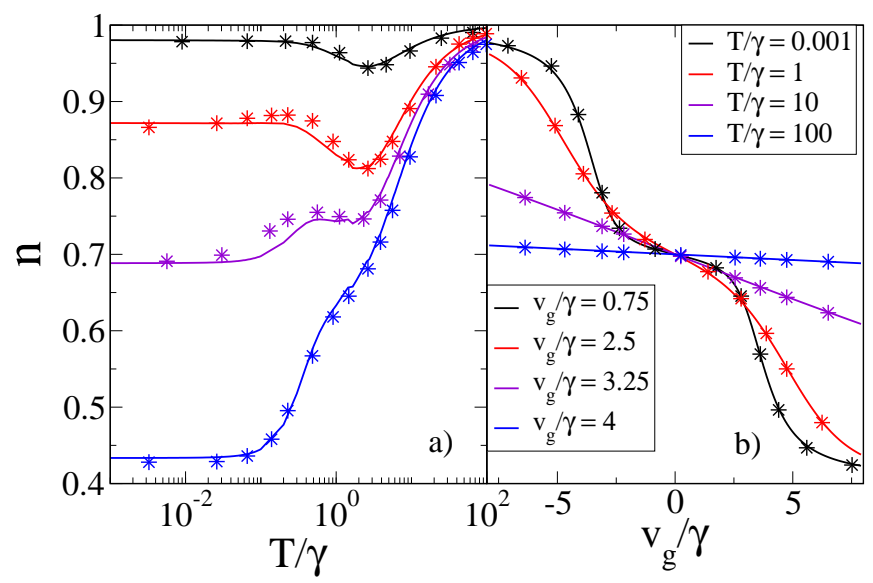

FIG. 3. Panel a) Densities as function of temperature for different gate voltages. Panel b) Densities as function of gate voltage for different temperatures. In both panels i-DFT results are compared with the NRG results of Ref. 36 for $U / \gamma=8$.

expression obtained in Ref. $26 \cdot \frac{35}{3}$

Kondo regime - So far we have constructed functionals in the temperature regime of Coulomb blockade $T \gtrsim T_{K}$ where $T_{K}$ is the Kondo temperature. In order to extend the range of applicability of our approximation to temperatures below $T_{K}$, we follow the ideas outlined in Ref. 27. There the central observation was that at zero temperature the correct behaviour of the zero-bias conductance (Eq. (4) ) is already contained in the KS conductance $G_{s}$ due to the Friedel sum rule ${ }^{37-39}$ Therefore, at zero temperature the derivative $\partial V_{\mathrm{xc}} / \partial I$ has to vanish at $I=0$. Following Ref. 27, we modify our functional as

$$
\begin{gathered}
v_{H x c}=[1-k(n, I, T)] \tilde{v}_{H x c}+k(n, I, T) v_{H x c}^{(0)}(n) \\
V_{x c}=[1-k(n, I, T)] \tilde{V}_{x c}(n, I, T)
\end{gathered}
$$

where $v_{H x c}^{(0)}$ is the zero-temperature, equilibrium Hxc potential of Ref. 38 which accurately parametrizes density matrix renormalization group results. We further introduce the prefactor $k(n, I, T)$ with the properties $k(n, I=0, T=0)=1$ and $\partial k(n, I, T) /\left.\partial I\right|_{I=0, T=0}=0$. The first property ensures that at zero current and zero temperature $v_{\mathrm{Hxc}}$ reduces to $v_{\mathrm{Hxc}}^{(0)}$, the second one leads to a vanishing correction to the $\mathrm{KS}$ zero-bias conductance at zero temperature. To be specific, we choose $k(n, I, T)=a(n, I) z(T)$, where $a(n, I)$ is the same prefactor used in Eq. (12) of Ref. 27. This prefactor, although combined with a different form for the Coulomb blockade functionals $\tilde{v}_{\mathrm{Hxc}}$ and $\tilde{V}_{\mathrm{xc}}$ ensures a good description of the finite bias conductance for relatively low temperatures. We also found it convenient to introduce another prefactor $z(T)=\left(1+(2.5 T / \gamma)^{3}\right)^{-1}$ to ensure a smooth transition to the Coulomb blockade form of the functional at high temperatures. Finally, we redefine $\eta=0.1 U / \gamma+0.36$ entering in the effective temperature

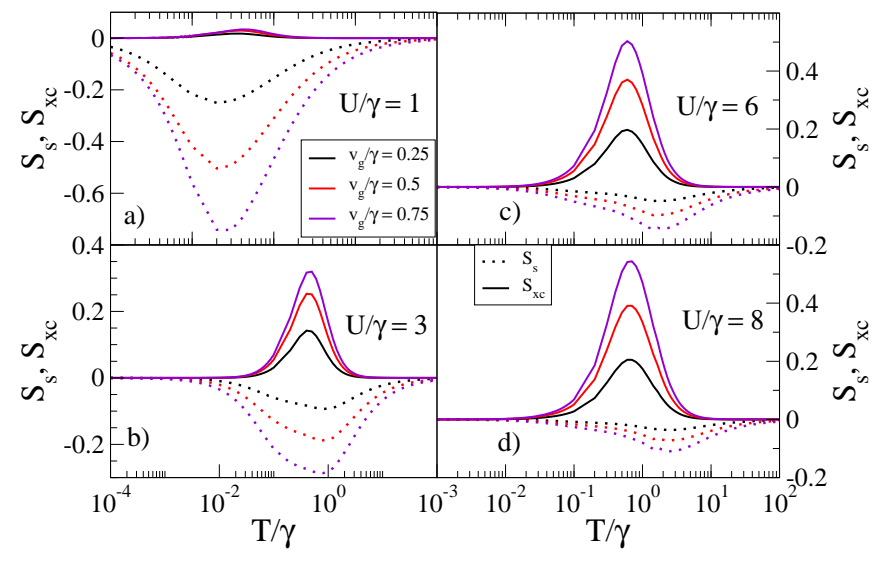

FIG. 4. Linear KS Seebeck coefficient $S_{s}$ and xc correction $S_{\mathrm{xc}}$ as function of temperature for different gate voltages and correlation strengths $U / \gamma$.

$T^{*}$ of Eq. (17) in order to correct the effect of the interactions at low temperatures. This is somewhat similar to Ref. 27 where the smoothening of the step features in the Coulomb blockade part of the functional had to be modified in the Kondo regime $T \lesssim T_{K}$.

As a first test of this functional, we calculate selfconsistent densities at equilibrium. In Fig. 3 a) we plot the densities obtained for different gate voltages as function of the temperature of the leads $T=T_{L}=T_{R}$ for the strongly correlated case with $U / \gamma=8$ and compare with numerical renormalization group (NRG) results of Ref. 36. Instead, in Fig. 3 b) we show equilibrium densities as function of gate voltage for different temperatures. The agreement of our i-DFT densities with the NRG ones is excellent.

We now turn to the Seebeck coefficient. As first step we analyze the relative magnitude of the KS Seebeck coefficient $\left(S_{s}\right)$ and the xc correction (see Eq. 9) as a function of the gate voltage and the correlation strength. In Fig.4, we can see that, as expected, the xc contribution becomes dominant for almost any temperature as $U / \gamma$ increases from 1 (Fig. 4a) to 8 (Fig. 4 $\mathrm{d}$ ), but also for intermediate values, $U / \gamma=3$, the two terms have a comparable magnitude for any value of the gate voltage. Notice that, since both potential and temperature are evaluated in units of $\gamma, S$ and $S_{x c}$ are dimensionless. In Fig. 5 we compare our results with the NRG ones of Ref. 36 for fixed gate potential as a function of temperature. Again, similar to Fig. 4, the panels report calculated values from weak (a) to strong correlations (d) in the dot. As expected we find a very good agreement between i-DFT and NRG for $T \gtrsim T_{K}$. For lower temperatures, i-DFT shows small discrepancies with respect to the reference result which exhibits a different evolution of the local minimum of the Seebeck coefficient when increasing the interaction.

In Fig. 6. we show the Seebeck coefficient as function of the gate voltage for different values of the temperature and again compare with NRG results of Ref. 36 . As already noticed above, for low temperatures there are 


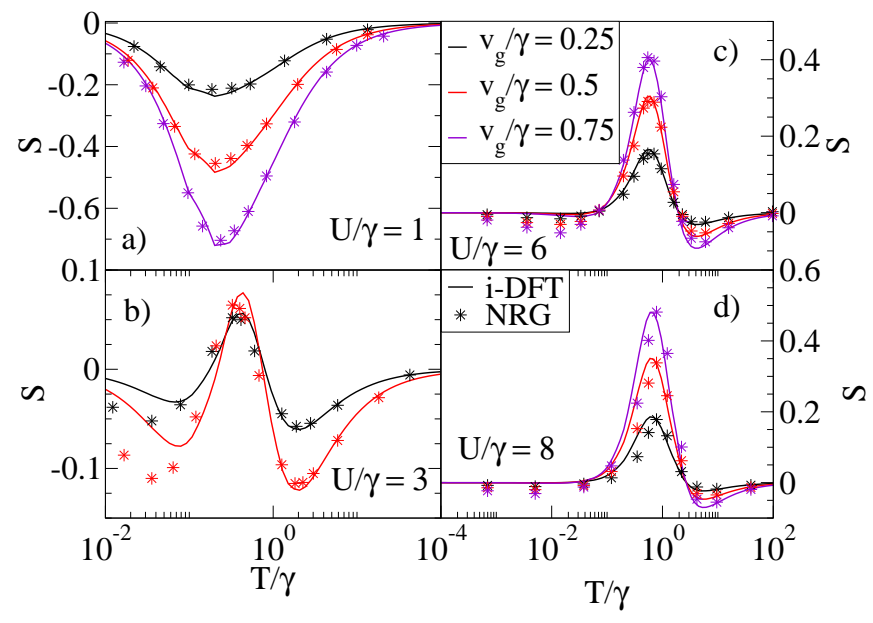

FIG. 5. Comparison of the Seebeck coefficient obtained with i-DFT with the NRG results of Ref. 36 as function of temperature for different gate voltages and correlation strengths $U / \gamma$.

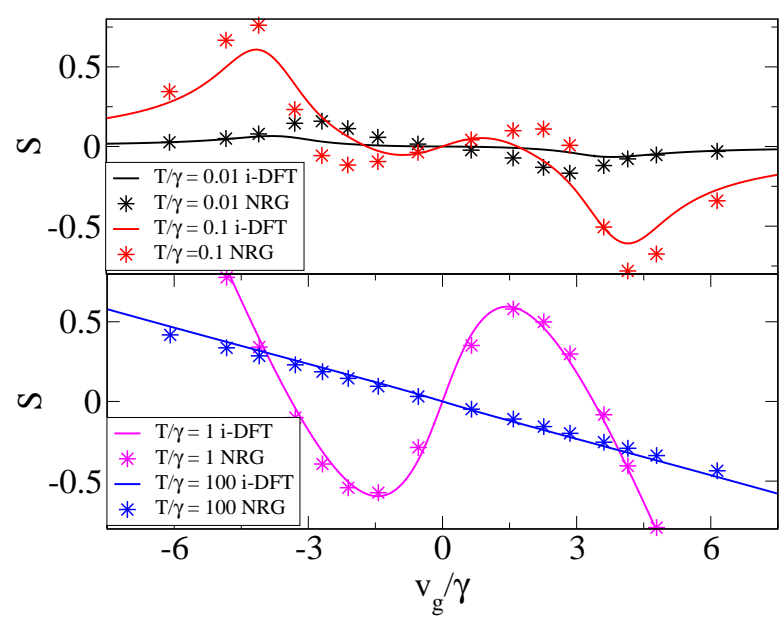

FIG. 6. Comparison of the Seebeck coefficient obtained with i-DFT with the NRG results of Ref. 36. $S$ is shown as function of the gate voltage for different temperatures and for strong correlations $U / \gamma=8$.

discrepancies at certain gate values although with our i-DFT approach we manage to obtain the qualitative behaviour of the NRG results. For $T / \gamma \gtrsim 1$, on the other hand, the i-DFT results are in excellent agreement with the NRG ones.

While the main focus of the present work is on the Seebeck coefficient, one can, of course, also calculate differential conductances from i-DFT. In Fig. 7, we show differential conductances for the SIAM at the particlehole symmetric point obtained with our present functional and compare them with those obtained using the functional of Ref. 27 as well as with functional renormalization group (fRG) results of Ref. 40. The i-DFT results with our present functional agree reasonably well with the reference fRG results although some details like the

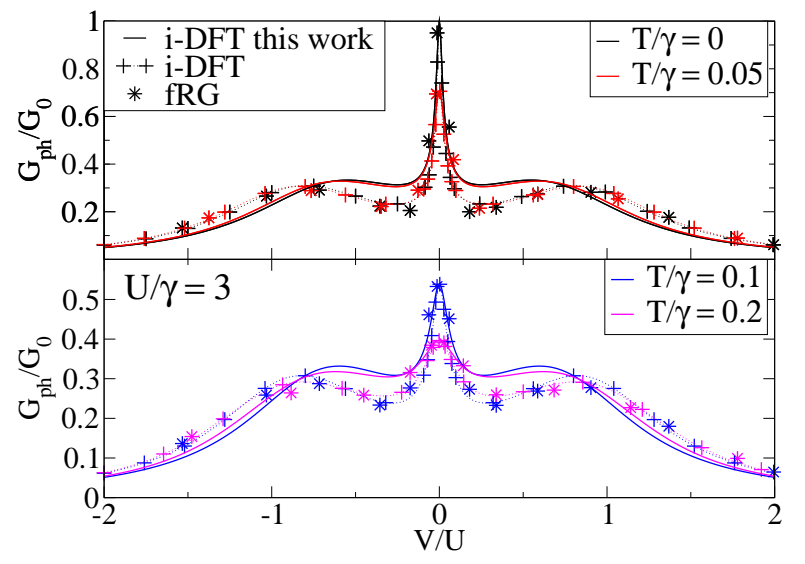

FIG. 7. Differential conductance $G_{\mathrm{ph}}$ at the particle hole symmetric point $v=-U / 2$ for the SIAM as function of bias $V$ for $U / \gamma=3$. The i-DFT results obtained with the functional of Eq. (19) are compared to those from Ref. 27 and the fRG results of Ref. 40. $G_{0}=1 / \pi$ is the quantum of conductance.

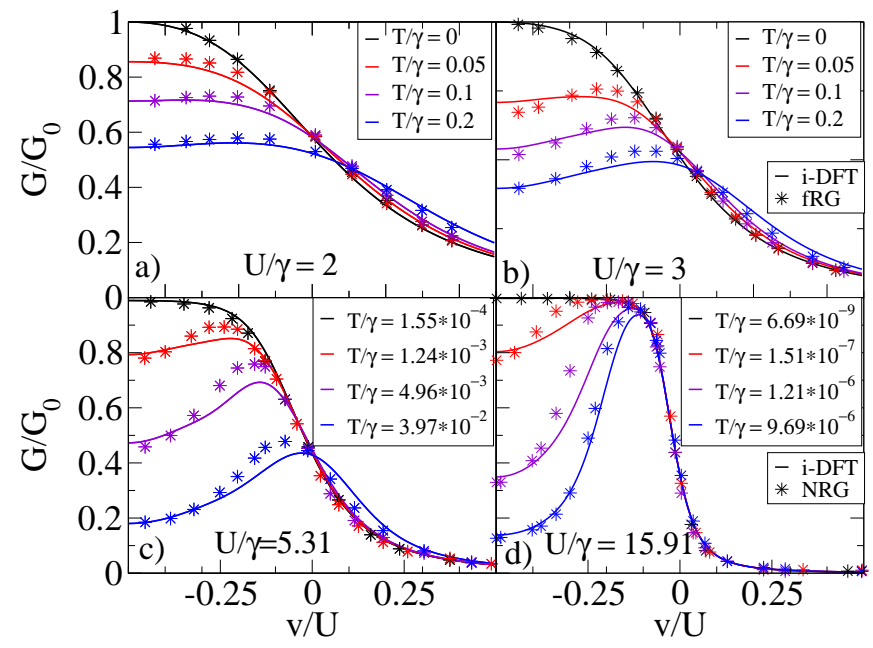

FIG. 8. Zero-bias differential conductance of i-DFT obtained with the functional of Eq. (19) as function of the gate level $v$ for different values of $U / \gamma$. In panels a) and b) the results are compared to fRG ones from Ref. 40, in panels c) and d) NRG results from Ref. 41 are used for comparison. $G_{0}=1 / \pi$ is the quantum of conductance.

overall shape of the side peaks seem to be better captured by the functional of Ref. 27. Finally, the differential conductance at zero bias has been calculated and compared with both fRG of Ref. 40 and NRG of Ref. 41 for different interaction strengths obtaining very good agreements, as can be appreciated in Fig. 8 .

\section{CONCLUSIONS}

In this work we have extended the recently proposed density functional framework for steady-state transport (i-DFT) to the situation when there is a temperature 
gradient across the junction. As a direct consequence, we have derived an exact expression for the Seebeck coefficient of the interacting system as a sum of the KS Seebeck coefficient and a correction term related to a derivative of the i-DFT xc bias functional.

For the SIAM we have constructed an approximation to the $(\mathrm{H}) \mathrm{xc}$ functionals both in the Coulomb blockade as well as in the Kondo regime. In the Coulomb blockade regime we found that both Hxc gate and xc bias potential consist of a sum or difference of two pieces, each of which depends only on the temperature of one of the leads. This property allows us to recover an earlier, approximate expression for the Seebeck coefficient in terms of purely DFT quantities 26 . We have compared the Seebeck coefficients for the SIAM obtained with our method with those from NRG calculations reported in the literature. While our parametrization by construction becomes exact at high temperatures $\left(T \gg T_{K}\right)$, in the Kondo regime $\left(T \ll T_{K}\right)$ the agreement is reasonable but not perfect. However, i-DFT is numerically much cheaper than more sophisticated many-body methods and extension to more complicated models can also be relatively straightforward.

\section{ACKNOWLEDGMENTS}

We gratefully acknowledge useful discussions with Florian Eich, David Jacob and Gianluca Stefanucci. We acknowledge funding by the grant "Grupos Consolidados UPV/EHU del Gobierno Vasco" (IT1249-19) as well as the grant of the "Ministerio de Economia y Competividad (MINECO)" (FIS2016-79464-P).

* nahualcsc@dipc.org

† roberto.dagosta@ehu.es

\$ stefan.kurth@ehu.es

1 C. B. Vining, Nature Mat. 7, 765 (2008).

2 C. B. Vining, Nature Mat. 8, 83 (2009).

3 Y. Dubi and M. Di Ventra, Rev. Mod. Phys. 83, 131 (2011).

4 H. J. Goldsmid, Introduction to thermoelectricity, Vol. 121 (Springer, 2010).

5 L. D. Hicks and M. S. Dresselhaus, Phys. Rev. B 47, 12727 (1993)

6 S. Datta, Electronic Transport in Mesoscopic Systems (Cambridge University Press, New York, 1997).

7 G. Cuniberti, G. Fagas, and K. Richter, eds., Introducing Molecular Electronics (Springer, New York, 2005).

8 J. C. Cuevas and E. Scheer, Molecular Electronics: An Introduction to Theory and Experiment (World Scientific, London, 2010).

9 C. W. J. Beenakker, Phys. Rev. B 44, 1646 (1991).

10 X. Zianni, Phys. Rev. B 78, 165327 (2008).

11 B. Sothmann, R. Sánchez, A. N. Jordan, and M. Büttiker, New J. Phys. 15, 095021 (2013).

12 R. Sánchez, B. Sothmann, A. N. Jordan, and M. Büttiker, New J. Phys. 15, 125001 (2013).

13 R. D'Agosta, Phys. Chem. Chem. Phys. 15, 1758 (2013)

14 F. G. Eich, A. Principi, M. Di Ventra, and G. Vignale, Phys. Rev. B 90, 115116 (2014).

15 P. Hohenberg and W. Kohn, Phys. Rev. 136, B864 (1964)

16 W. Kohn and L. J. Sham, Phys. Rev. 140, A1133 (1965).

17 M. Thoss and F. J. Chem. Phys. 148, 030901 (2018).

18 E. Runge and E.K.U. Gross, Phys. Rev. Lett 52, 997 (1984).

19 G. Stefanucci and C.-O. Almbladh, EPL 67, 14 (2004).

20 G. Stefanucci and C.-O. Almbladh, Phys. Rev. B 69, 195318 (2004).

21 N. Sai, M. Zwolak, G. Vignale, and M. Di Ventra, Phys. Rev. Lett. 94, 186810 (2005)

22 M. Koentopp, K. Burke, and F. Evers, Phys. Rev. B 73, 121403 (2006).

23 G. Stefanucci, S. Kurth, A. Rubio, and E.K.U. Gross, in Molecular and Nano Electronics: Analysis, Design, and Simulation, 17, edited by J. Seminario (Elsevier, Amsterdam, 2006).

24 G. Vignale and M. Di Ventra, Phys. Rev. B 79, 014201 (2009).

25 G. Stefanucci and S. Kurth, Nano Lett. 15, 8020 (2015)

${ }^{26}$ K. Yang, E. Perfetto, S. Kurth, G. Stefanucci, and R. D'Agosta, Phys. Rev. B 94, 081410 (2016).

27 S. Kurth and G. Stefanucci, Phys. Rev. B 94, 241103 (2016).

28 D. Jacob and S. Kurth, Nano Lett. 18, 2086 (2018).

29 Needless to say, we assume $T>\Delta T / 2$ such that $T_{L}, T_{R}>$ 0 .

30 S. Kurth, D. Jacob, N. Sobrino, and G. Stefanucci, Phys. Rev. B 100, 085114 (2019)

31 N. Dittmann, J. Splettstoesser, and N. Helbig, Phys. Rev. Lett. 120, 157701 (2018)

32 N. Dittmann, N. Helbig, and D. M. Kennes, Phys. Rev. B 99, 075417 (2019).

33 S. Kurth and G. Stefanucci, J. Phys. Condens. Mat. 29, 413002 (2017)

34 S. Kurth and G. Stefanucci, Eur. J. Phys. B 91, 118 (2018).

35 Notice that by comparing (18) and (9) one arrives at $S=$ $S_{s}-S_{x c}$. This reflects the standard definition $V_{x c}=V_{s}-V$.

36 T. Costi and V. Zlatić, Phys. Rev. B 81, 235127 (2010).

37 G. Stefanucci and S. Kurth, Phys. Rev. Lett. 107, 216401 (2011).

38 J. P. Bergfield, Z.-F. Liu, K. Burke, and C. A. Stafford, Phys. Rev. Lett. 108, 066801 (2012).

39 P. Tröster, P. Schmitteckert, and F. Evers, Phys. Rev. B 85, 115409 (2012).

40 S. G. Jakobs, M. Pletyukhov, and H. Schoeller, Phys. Rev. B 81, 195109 (2010).

41 W. Izumida, O. Sakai, and S. Suzuki, J. Phys. Soc. Jpn 70, 1045 (2001). 